\title{
Arrhythmogenic right ventricular cardiomyopathy/ dysplasia clinical presentation and diagnostic evaluation: Results from the North American Multidisciplinary Study
}

\section{Citation}

Marcus, Frank I., Wojciech Zareba, Hugh Calkins, Jeffrey A. Towbin, Cristina Basso, David A. Bluemke, N.A. Mark Estes, et al. 2009. “Arrhythmogenic Right Ventricular Cardiomyopathy/ dysplasia Clinical Presentation and Diagnostic Evaluation: Results from the North American Multidisciplinary Study." Heart Rhythm 6 (7) (July): 984-992. doi:10.1016/j.hrthm.2009.03.013.

\section{Published Version}

doi:10.1016/j.hrthm.2009.03.013

\section{Permanent link}

http://nrs.harvard.edu/urn-3:HUL.InstRepos:29048917

\section{Terms of Use}

This article was downloaded from Harvard University's DASH repository, and is made available under the terms and conditions applicable to Other Posted Material, as set forth at http:// nrs.harvard.edu/urn-3:HUL.InstRepos:dash.current.terms-of-use\#LAA

\section{Share Your Story}

The Harvard community has made this article openly available.

Please share how this access benefits you. Submit a story. 


\title{
Arrhythmogenic Right Ventricular Cardiomyopathy/Dysplasia, Clinical Presentation and Diagnostic Evaluation: Results from the North American Multidisciplinary Study
}

\author{
Frank I Marcus, MD, Wojciech Zareba, MD, Hugh Calkins, MD, Jeffrey A Towbin, M.D., Cristina \\ Basso, MD, David A Bluemke, MD, PhD, N. A. Mark Estes III, MD, Michael H Picard, M.D., \\ Danita Sanborn, MD, Gaetano Thiene, MD, Thomas Wichter, MD, David Cannom, MD, David \\ J Wilber, MD, Melvin Scheinman, MD, Henry Duff, MD, James Daubert, MD, Mario Talajic, \\ MD, Andrew Krahn, MD, Michael Sweeney, MD, Hasan Garan, MD, Scott Sakaguchi, MD, \\ Bruce Lerman, MD, Charles Kerr, MD, Jack Kron, MD, Jonathan Steinberg, M.D., Duane \\ Sherrill, PhD, Kathleen Gear, RN, Mary Brown, MS, Patricia Severski, BS, Slava Polonsky, \\ MS, and Scott McNitt, MS
}

\begin{abstract}
Background-Prior reports on patients with ARVC/D focused on individuals with advanced forms of the disease. There are limited data regarding diagnostic performance of various testing modalities in newly identified individuals suspected of having ARVC/D.

Objectives-The Multidisciplinary Study of Arrhythmogenic Right Ventricular Cardiomyopathy/ Dysplasia (ARVC/D) was initiated to study the clinical characteristics and the diagnostic evaluation of a large group of newly identified patients with ARVC/D.
\end{abstract}

\begin{abstract}
Methods-A total of 108 newly diagnosed patients with suspected ARVC/D were prospectively enrolled in the United States and Canada. The patients underwent noninvasive and invasive tests using standardized protocols that were initially interpreted by the enrolling center and adjudicated by blind analysis in six core laboratories. The patients were followed for a mean of $27 \pm 16$ months (0.2 - 63 months).
\end{abstract}

\begin{abstract}
Results-The clinical profile of these newly diagnosed patients differs from those reported with more advanced disease. There was considerable difference in the initial and final classification of the presence of ARVC/D after the diagnostic tests were evaluated by the core laboratories. Final clinical diagnosis was 73 affected, 28 borderline, and 7 unaffected. Individual tests agreed with final diagnosis in $50 \%$ to $70 \%$ of the 73 who had final classification as affected.
\end{abstract}

\begin{abstract}
Conclusions-The clinical profile of 108 newly diagnosed probands with suspected ARVC/D indicates that a combination of diagnostic tests is needed to evaluate the presence of right ventricular structural, functional and electrical abnormalities. Echocardiography, RV angiography, SAECG and Holter monitoring provide optimal clinical evaluation of patients suspected of ARVC/D.
\end{abstract}

(C) 2009 The Heart Rhythm Society. Published by Elsevier Inc. All rights reserved.

Corresponding Author: Frank I Marcus, MD, University of Arizona, Section of Cardiology, 1501 N. Campbell Avenue - Cardio Rm. 6304, Tucson, AZ 85724-0001, fmarcus@email.arizona.edu.

Publisher's Disclaimer: This is a PDF file of an unedited manuscript that has been accepted for publication. As a service to our customers we are providing this early version of the manuscript. The manuscript will undergo copyediting, typesetting, and review of the resulting proof before it is published in its final citable form. Please note that during the production process errors may be discovered which could affect the content, and all legal disclaimers that apply to the journal pertain.

No conflict of interest 


\section{Keywords}

Cardiomyopathy; ventricular tachycardia; Genetics; Implantable Cardioverter Defibrillator

Arrhythmogenic Right Ventricular Cardiomyopathy/Dysplasia (ARVC/D) is an inherited cardiomyopathy characterized by fibro-fatty replacement primarily of right ventricular muscle. The resultant heterogeneous structure of the right ventricular myocardium results in ventricular arrhythmias, including premature ventricular contractions (PVCs), and non-sustained or sustained ventricular tachycardia. Sudden death due to ventricular and structural or functional alterations of the right and left ventricles may lead to congestive heart failure. (1-3) ARVC/D is uncommon, but may account for up to $20 \%$ of cases of sudden death among young individuals. (4-7)

The first clinical profile of ARVC/D was published in 1982.(8) In that report, 24 cases of ARVC/D were described, almost all of whom had advanced disease and were referred to a tertiary medical center for the treatment of recurrent ventricular tachycardia. The structural and functional changes of the right ventricle were readily apparent. Since then, it has become evident that the disease can be exceedingly difficult to diagnose, particularly when there are minimal structural and/or functional alterations of the right ventricle. (9) Recognition of the problems in diagnosing ARVC/D and the fact that there is no "gold standard" or single test that is diagnostic of ARVC/D led to the formation of a task force that forth major and minor criteria in 1994 to aid in the diagnosis. (10) Despite these standardized diagnostic guidelines, there remains uncertainty, primarily due to lack of quantification of many of these task force criteria. In addition, the clinical profile of a large number of newly diagnosed patients with ARVC/D, who were systematically evaluated using standardized protocols to determine the presence of task force criteria, has not been reported previously.

The Multidisciplinary Study of ARVC/D was initiated in 2001 with the following goals: 1) to study a large group of newly diagnosed patients with this uncommon disorder, 2) to elucidate the genetic etiology of this disease, 3) to evaluate treatment of ARVC/D in these patients. This report focuses on the diagnostic and clinical features of the first 108 unrelated probands who were enrolled in this study. Comparison and relative contribution of different testing modalities is emphasized

\section{Methods}

\section{Organizational Structure}

The Multidisciplinary Study of Right Ventricular Cardiomyopathy/Dysplasia established the North American ARVC/D Registry consisting of 18 enrolling centers in the United States and Canada (see appendix), a clinical center at the University of Arizona, a data coordinating center at the University of Rochester, a genetic center at Baylor College of Medicine, six core laboratories in the United States and Europe, and an NIH appointed Data and Safety Monitoring Board (see appendix). The design of the study has been published previously. (11)

\section{Patient Population}

Patients, 12 years of age or older and newly diagnosed with ARVC/D, were evaluated for enrollment. The sequence of evaluation is shown in Figure 1. Initially patients were excluded if they had an ICD implanted prior to enrollment. Since patients and their personal physicians often became aware of this study after ICD implant, this severely hampered referral for enrollment. During the second year of the study, the Data Safety Monitoring Board agreed to allow enrollment of patients whose ICDs were implanted within 6 months. In the last two years 
of the study, patients were permitted to be enrolled if they had an ICD implanted for less than two years. All subjects signed institutional review board approved informed consent.

\section{Evaluation and clinical testing}

The standardized tests included a 12 lead electrocardiogram (ECG), signal-averaged ECG (SAECG), 24 hour Holter monitor and electrophysiological study with programmed stimulation for induction of ventricular arrhythmias. The SAECG was classified as positive if 2 of the 3 parameters were abnormal. (12) The electrophysiology study protocol was based on that used in the MUSTT trial. (13) Imaging of the right ventricle was done by echocardiography, (14) magnetic resonance imaging (MRI) (15) and right ventricular angiography. Right ventricular free-wall biopsies were obtained and sent to the core laboratory for pathological examination including histomorphometric analysis. $(16,17)$ Septal biopsy samples were separated from the free wall biopsies and sent to the genetic lab for viral studies. Blood was obtained for genetic analysis. Standardized protocols for the performance of these diagnostic tests were developed and distributed to the enrolling centers. The ECG, SAECG, echocardiogram, and MRI protocols are on the www.arvd.org website.

At the time of screening for enrollment, copies of the ECG, SAECG and reports of the imaging test results from the referring and enrolling centers were sent to the principal investigator (FM) for evaluation for enrollment based on the task force criteria. (Figure 1). In order to enhance accuracy as well as uniformity of interpretation of the diagnostic studies, core laboratories were established. The diagnostic tests were evaluated blindly by the core laboratories. The internal consistency of the intraobserver variability of the imaging studies was excellent with intraclass correlation of $89 \%$ for echocardiogram, and $93 \%$ for MRI. The rating reliability was assessed by comparing the variability of different ratings of the same subject to the total variation across all ratings and all subjects.

The diagnostic test results were sent to the data coordination center and entered into a secure web based data management system. Based on the core lab interpretation of each test as affected, borderline, or non affected, the principal investigator (FM) performed final classification of the phenotype. Affected probands met task force criteria of 2 major, 1 major and 2 minor or 4 minor criteria; borderline probands had either a major criterion and one minor or three minor criteria. Blood for DNA extraction and lymphoblastoid cell line immortalization was obtained from all but nine of the probands.

\section{Statistical analysis}

Descriptive characteristics of the probands are presented as means and standard deviations and proportions stratified by phenotypic classification, $\mathrm{p}$ values were obtained by Pearson's chi square test. To determine the relative contribution of each diagnostic procedure we utilized the c statistic, a standard measure of discriminative power for logistic regression models. Each diagnostic measure phenotype was dichotomized as 1 for affected and 0 otherwise. The seven different core lab test results (ECG, SAECG, Holter, echocardiogram, angiogram, MRI, and biopsy) were entered into a multivariate logistic regression model to capture the overall prediction. The model was run seven times, each time leaving out a different diagnostic measure. The differences in $\mathrm{c}$ statistics between the "full" model and each model that withheld a different diagnostic test were plotted to show the relative contribution to model fit which each made. In addition, a comparison between models containing all seven tests were compared to models containing decreasing number of tests starting with 6, 5, 4 using the likelihood scores. 


\section{Results}

\section{Demographic and clinical characteristics (Table 1)}

The database was evaluated as of October 4, 2007 at which time final classification was available in 112 probands. Of these, four patients diagnosed as ARVC/D were subsequently documented to have cardiac sarcoidosis by pathological examination of myocardial or cervical lymph node biopsy even though they fulfilled task force criteria. Therefore, for descriptive purposes of the ARVC/D population, these 4 patients were excluded and the analysis was performed on 108 probands. The final classification was 73 affected, 28 borderline and 7 unaffected.

Of the 108 probands, $57 \%(62 / 108)$ were male. The ethnic distribution was as follows: 95 Caucasian, 4 Hispanic, 7 Asian, and 2 African-American. Probands ranged in age at diagnosis from 12 to 63 years of age. The mean age at onset of symptoms was $36 \pm 15$ years. The mean age at diagnosis was $38 \pm 13$ years, and nine were diagnosed between the ages of 12 to 18 . The mean age at enrollment was $39 \pm 14$ years, range 15 to 78 years of age. Four probands had NYHA Class II and one had class IV right heart failure. Palpitation was the most common symptom and was present in 56\% (61/108) of enrolled probands, followed in frequency by dizziness in 27\% (29/108) and syncope in 21\% (23/108). The most frequent reason for the clinical suspicion of ARVC/D was a history of ventricular arrhythmias reported in $70 \%$ (76/108). One proband had a cardiac arrest as the first symptom. Of particular interest is that $34 \%$ of the probands participated in competitive or professional sports prior to diagnosis. Of these, $68 \%$ were male. An additional $36 \%$ of patients were active in recreational sports.

\section{ECG findings}

One of 108 ECGs was uninterpretable due to atrial fibrillation with a ventricular paced rhythm; 11 had complete right bundle branch block (RBBB) and one had left bundle branch block. Therefore, 95 ECGs were analyzed. Thirty probands $32 \%$ (30/95) had T wave inversion beyond V3; their final phenotype was 29 affected and 1 borderline. Three probands had $\mathrm{T}$ wave inversion limited to leads $\mathrm{V}_{4}, \mathrm{~V}_{5}$, or $\mathrm{V}_{6}$. It was also observed that $\mathrm{T}$ wave inversion was present in two or three of the inferior leads, II, III and AVF in 20 probands, 14 of whom had T wave inversion beyond $\mathrm{V}_{3}$ in the precordial leads. Therefore, this finding correlated with the extent of the precordial $\mathrm{T}$ wave inversion.

There were 5\% (5/95) who met the task force criteria of QRS $>110$ msecs in $V_{1}, V_{2}$ and $V_{3}$. Eighteen had QRS > 110 msecs in one, two or all of the precordial leads $V_{1}-V_{6}$. Of the 86 patients who had 24 hour Holter recordings, 57\% (49/86) had >1000 PVCs/24 hours; of whom $6 \%(5 / 86)$ had greater than 10,000 PVCs in 24 hours. There were $16 \%$ who had <200 PVCs/ 24 hours, and 20\% had between 200 and 1000 PVCs/24 hours. The morphology and axis of the QRS complex during clinical VT could be determined in 63\% (24/38) of probands who presented with clinical sustained VT. Ten had reports or tracings of LBBB inferior axis and 14 of LBBB superior axis. Eight reported LBBB VT, axis undetermined and one had sustained RBBB VT. Five had inadequate documentation to determine the morphology of the VT. The rate of the clinical VT was documented in 14 at a mean of $215 \pm 21$ beats per minute. Of the 38 patients with clinical sustained VT, 59\% (19/32) were inducible at EPS. Nine of these 19 had the same morphology induced as their clinical VT.

\section{Cardiac Imaging}

The imaging test interpreted by the core laboratory that reported the most severe abnormality was used for classification of structural and functional abnormalities. Severely reduced right ventricular wall motion or global function was reported in $36 \%$ by MRI, in $15 \%$ by echocardiography and $21 \%$ by right ventricular angiogram. (Table 2 ) 
There were 38 probands phenotyped as affected who had both echocardiograms and MRI studies that were suitable for analysis of RV and LV function by the core laboratories. The echocardiograms were interpreted as showing normal RV function (FAC $\geq 32 \%$ ) in $58 \%$ while the MRI reported fewer probands with normal RVEF $(\geq 52 \%)$ in $34 \%$. Conversely the MRI reported more probands with moderate or severely reduced RV function (RVEF $\leq 45 \%)$ in $32 \%$ than did the echocardiogram $(\mathrm{FAC} \leq 26 \%)$ in $16 \%$. All but $2 / 75$ probands had normal or mildly decreased LV function by echocardiography. Of 38 probands phenotyped as affected and who had all three imaging tests, the agreement between all three interpretable imaging tests classified as affected was 45\% (17/38) and 32\% (12/38) for those with two imaging tests in agreement. Ten of 108 probands had only one interpretable imaging test.

Table 3 shows the frequency of diagnostic tests by core lab analysis as affected, borderline or unaffected in the 73 probands with final classification as affected and in the 28 probands with final classification as borderline based on the task force criteria. This provides an estimate of the percentage of each test that one may expect to be positive (affected), borderline or non affected (negative or normal).

\section{Relation of initial classification versus final classification after core laboratory analysis of data}

The design of this study provided a unique opportunity to compare the extent of agreement in the interpretation of imaging studies (echocardiogram, right ventricular angiogram and magnetic resonance imaging) by the referring centers with that of the core laboratories where the studies were interpreted blindly. The following criteria were utilized. When the referring center described only a minor task force imaging abnormality, e.g. mild hypokinesis, the imaging study classification was determined to be borderline. When a major task force imaging criterion was selected, e.g. severe global dysfunction, the imaging study was classified as affected. This information was compared with the blinded analysis reported by the core laboratory as to whether the imaging study was normal, borderline or unaffected.

The frequency of a positive test for ARVC/D by MRI was considerably greater at the referral center than that by the core laboratory. Of the 41 MRIs thought to have a major imaging abnormality by the referring center, $29 \%$ of those were not confirmed by the MRI core laboratory. The interpretation of abnormalities of 2D echocardiogram showed the reverse trend; i.e. a smaller percent of studies was thought to be affected by the referring center than by core laboratory analysis. There were 26 echocardiograms categorized as affected by the referring center as compared with the core laboratory analysis of 45 affected. Right ventricular angiography interpretation was similar between the referring center and the core laboratory.

The relation of the initial classification at enrollment based on data from the referring center and the final classification after core lab analysis is of interest. Of the 108 probands, 86 probands were classified as affected on enrollment. After blind interpretation of diagnostic testing by core laboratories $78 \%$ (67/86) had the final classification as affected, 17 were borderline, and 2 were unaffected. (Figure 2A) Nineteen probands met some but not all of the task force criteria and were enrolled as borderline. Only 26\% (5/19) of those classified as borderline at enrollment met task force criteria after final classification. $(\mathrm{p}=.001)$. (Figure 2B) Thus if the task force criteria were present at enrollment, there is a reasonable certainty that the patient would have a final classification as affected whereas there was a lower chance that the suspected probands who were "borderline" at enrollment would meet the established task force criteria. 


\section{Diagnostic performance of individual tests and their combination to the final diagnosis}

We performed comprehensive analyses to evaluate the diagnostic performance of the following core lab tests performed in this study: ECG, SAECG, Holter, echocardiogram, RV angiogram, MRI, and RV biopsy. Table 3 and Figure 3 shows the frequency of diagnostic tests by core lab analysis as affected, borderline or unaffected in the 73 probands with final classification as affected and in the 28 probands with final classification as borderline based on the task force criteria. This provides an estimate of the percentage of each test that one may expect to be positive (affected), borderline or non affected (negative or normal). Figure 4 shows diagnostic performance of individual tests evaluated separately without taking into account other tests. The echocardiogram, RV angiogram and ECG show a better diagnostic performance than other evaluated tests.

We also performed analyses of the diagnostic performance of the above tests when evaluated simultaneously, reflecting clinical practice when physicians obtain results of several tests simultaneously. (Table 4) We used all seven tests to determine optimal categorization of patients and then we removed each test one at a time from this 7-test model to determine the decline in the c-statistics reflecting diagnostic performance of a given test. Figure 4 demonstrates that the biggest decline in the diagnostic performance of the model is observed when the echocardiogram is removed. Removing RV angiogram and SAECG one at a time also caused a decline in the performance of the testing. On the other hand, removing the MRI, ECG, and RV biopsy, one at a time, caused less decline in the predictive model.

Additional analyses were performed to evaluate how the models with less than the full set of 7 tests would perform in diagnosing affected ARVC/D probands. The best 6-variable model that performed as well as the full 7-variable model consisted of all tests except the MRI. When a 5-variable model was considered, the best performance was achieved when echocardiogram, RV angiogram, ECG, SAECG, and Holter were used and MRI and RV biopsy were removed. This model was only marginally inferior to 7-variable model. When using 4- and 3-variable models the diagnostic performance of testing was significantly compromised. Based on the analyses of the models we concluded that evaluating RV echocardiogram, RV angiograms, and SAECG is optimal for all successful models. Routinely used ECG and Holter tests complement these tests providing best diagnostic performance.

\section{Genetic data}

Single or digenic mutations were found in 33 of 100 probands ( 8 probands did not provide blood for genetic analysis). Plakophilin-2 (PKP-2) was the most commonly found gene variant, present in 22\% (22/100). Six of the 22 probands with PKP-2 mutations had compound heterozygosity with mutations in other desmosomal proteins, one with three variants. Of the remaining 11, two had heterozygous mutations in desmosomal genes other than PKP-2, seven had homozygous mutations in other desmosomal proteins and two had a polymorphism variant. There was a similar frequency of desmosomal abnormalities in approximately one third of those phenotyped borderline and of those phenotyped affected. This observation supports the hypothesis that most if not all of the probands phenotyped as borderline do have this disease. This illustrates the relative insensitivity of the task force criteria for the diagnosis of early disease.

\section{Discussion}

This is the first study of a large number of newly diagnosed patients, suspected of having ARVC/D, who were studied systematically with a variety of standardized diagnostic tests. The clinical characteristics of this large group of newly diagnosed probands provide a unique profile of these patients. In addition, this design allows, for the first time, a comparison of the 
interpretation of the three imaging tests by the referring center with blinded interpretation of the tests by the core laboratories. It also permits comparison of the interpretation of the imaging tests with one another. The initial classification of whether the patient met the task force criteria was compared with final classification based on the interpretation of the diagnostic tests by the core laboratories (ECG, echocardiogram, MRI, RV angiogram, pathology). These study results complement the clinical profile of 69 living patients with ARVC/D reported by Dalal et al.

The clinical characteristics of the 108 probands indicate that there is a slight preponderance $57 \%$ (57/108) of men. The major reason for the clinical suspicion of ARVC/D was a recent history of ventricular arrhythmias of which $35 \%$ were reported as sustained VT. The finding that $34 \%$ of the probands were competitive or professional athletes lends support to the hypothesis that vigorous and/or sustained athletic activity may facilitate the phenotypic expression of the disease due to repetitive stretch of the vulnerable thin walled right ventricle with a genetic desmosomal protein abnormality that is the underlying myocardial defect responsible for this disease. (19-21)

It is of interest that 29 of 30 probands with $\mathrm{T}$ wave inversion beyond $\mathrm{V} 3 \mathrm{had}$ a final phenotype as affected. Only $17 \%$ (12 affected, 3 borderline, 1 unaffected) of the ECGs in probands met the criteria proposed by Peters et al of QRS duration of $\mathrm{V}_{1}+\mathrm{V} 2+\mathrm{V}_{3} / \mathrm{V}_{4}+\mathrm{V}_{5}+\mathrm{V}_{6}$ of $\geq 1.2$. (22) We also analyzed digitized ECGs using the ratio of QRS duration of V2/V5. We observed that $27 \%$ had a ratio of $\geq 1.2$. Another manifestation of localized prolongation of the QRS in right precordial leads is that of an $\mathrm{S}$ wave duration of $\geq 55$ msecs which was found in $95 \%$ by Nasir et al (23), who defined the $S$ wave duration as the interval from the nadir of the $S$ wave until the end of the QRS complex, excluding any R prime wave. We observed this finding in $\mathrm{V}_{1}, \mathrm{~V}_{2}$, or $\mathrm{V}_{3}$ in only $32 \%$ when the $\mathrm{R}$ prime was included. Cox, et al measured the time from the nadir of the $\mathrm{S}$ wave to the end of all depolarization, including $\mathrm{R}$ prime and any epsilon wave, and found a $S$ wave $>55$ msecs in $71 \%$. (24) In our series, the one patient with an epsilon wave also had RBBB. Two probands with sarcoidosis, not included in this analysis, also had epsilon waves. The SAECG was positive ( 2 of 3 criteria) in $58 \%$ of probands. The lower incidence of several of the ECG findings in our study as compared to those in the literature may reflect the fact that the population in this study is relatively newly diagnosed and therefore likely to have earlier manifestations of this disease.

Recent evidence indicates that patients with desmosomal genetic abnormalities may have major or predominant decrease in LV function that is greater than the decrease in RV function. (25, $26,27)$ The fact that only 2 patients had moderate decrease in LVEF (35\% and 40\%) may be due to selectively not enrolling those patients with ARVC/D with severe LV impairment since the task force criteria indicates that only patients with no or mild LV impairment should be considered to have this diagnosis.

Of particular importance are the data indicating that diagnostic classification based on referral center interpretation of test results may not be confirmed after independent review of the tests, particularly the imaging studies. For example, of the 86 probands who met task force criteria based on initial interpretation of diagnostic tests at the referring center, reclassification after core laboratory analysis, indicated that $19(23 \%)$ did not meet the established task force criteria and received a final classification as borderline and 2 patients were classified as unaffected.

Also, five of the 19 probands initially classified as borderline had final classification as affected. This brings into question the reliability of diagnostic tests as currently performed and interpreted for the diagnosis of ARVC/D. The observation that the frequency of a positive test for ARVC/D by MRI is considerably greater at the referral center than that of the core laboratory is particularly striking. Of the 41 MRIs thought to have a major imaging abnormality by the referring center, only $63 \%$ of these were confirmed by the MRI core laboratory. This 
finding is consistent with a previous report that documented the apparent high false-positive rate of the interpretation of the MRI for the diagnosis of ARVC/D by referring centers. (9)

There are several possible reasons for the discrepancy in interpretation of the imaging studies. $\mathrm{ARVC} / \mathrm{D}$ is a rare disease that requires specific protocols for optimum evaluation. The imaging core laboratories used a consistent definition that, whenever possible, incorporated quantitative criteria. The MRIs from the referring centers did not always contain short axis images that were required in the standardized protocol; quantitative measurements of RV dimensions could not be made in 20\%. Physicians who interpret the MRI may have limited experience in differentiating normal structure and function of the highly irregular contour of the right ventricle from that of $A R V C / D$, especially in the early stages of the disease. It has been documented that there is a high rate of error in differentiating normal RV wall motion from abnormal by MRI, particularly near the insertion of the moderator band.(28) In addition, there is substantial error in the interpretation of fatty infiltration as well as thinning of the right ventricular wall.(29) It is not well appreciated that, in the normal heart, a certain amount of fatty tissue is usually present within the myocardium of the right ventricular wall, particularly in the anterolateral and apical regions. $(30,31)$ These findings should be taken into account when interpreting structural abnormalities in patients suspected of ARVC/D. This emphasizes the importance of expert interpretation of the complex shaped RV and the need for quantitation of the RV structure and function. $(32,33)$ This is beginning to be addressed as a result of this study. (34)

Viral analysis of endomyocardial biopsies were performed because of uncertainty as to the role of viral myocarditis as a cause of ARVC/D in some cases and/or that ARVC/D may predispose to viral myocarditis which could lead to decrease in RV function and accelerate the progression of the disease. (35-38)

Evidence of viral infection was found in $15 \%$ of endomyocardial biopsies. Parvovirus was detected in 4 probands. Interestingly, enterovirus, present in five of twelve patients with ARVC/ $\mathrm{D}$ in a previous study, was not identified in this population. This could be due to a change in the epidemiology of viral diseases. The relatively small percent of positive viral identifications does not support a primary role for viral myocarditis as an etiology of ARVC/D. However, it does not exclude the possibility that ARVC/D may predispose to secondary viral myocarditis in a subset of patients or that a viral infection could unmask the disease. This could lead to a more rapid progression of the disease and possibly precipitate ventricular arrhythmias in ARVC/D.

Endomyocardial biopsy was performed in 59\%. This was the lowest percent of any of the diagnostic tests. (Table 1) An average of three samples were obtained per person, range one to seven biopsy samples. Nine of the biopsies were obtained from the septum. This may have been due to concern among the investigators of the risk of the procedure although the protocol indicated that the biopsy be obtained from the free wall of the right ventricle. The reason for not targeting the septum is that the septum is seldom involved in the disease process.(17) The safety of right ventricular free wall biopsy was demonstrated in other series since there was no instance of cardiac tamponade. $(39,40)$ In our experience one patient had a small pericardial effusion after biopsy that resolved spontaneously. It should be noted that, at the onset of the study, interventionalists in each enrolling center were taught how to safely perform the endomyocardial biopsy by video and by written instructions from the director of the angiography core laboratory. An unexpected finding was the presence of sarcoidosis identified by myocardial biopsy in 3 probands. In a fourth patient sarcoidosis was suspected because of the pulmonary infiltrates and the diagnosis was confirmed by cervical biopsy. This indicates that patients with sarcoidosis can have signs and symptoms that mimic ARVC/D. This finding of sarcoidosis masquerading as ARVC/D has been reported previously. (41) 
The analysis of the diagnostic performance of individual tests favors echocardiogram, RV angiogram, SAECG, and ECG. When evaluating them in combination, it is recommended that a minimum of the following 5 tests be performed: echocardiogram, RV angiogram, SAECG, ECG, and Holter. In our cohort of newly identified patients the diagnostic performance of MRI and RV biopsy seems to be inferior in comparison to the above five tests. However, it should be noted that the number of patients with MRI and RV biopsy was less than the other tests and this might have contributed to the underperformance of these studies. Further comparative analyses in a homogenous patient population using standardized core lab evaluated tests are needed to determine whether these findings will be confirmed.

The question arises as to whether the probands with final phenotype as borderline do indeed have ARVC/D. Probands with final classification as affected had slightly more symptoms of dizziness, nausea, fatigue, palpitations and chest pain than patients classified as borderline. Borderline probands had nearly the same incidence of syncope (25\%) and history of no ventricular tachycardia (25\%) as affected probands (21\% and 19\%). Affected probands had sustained VT induced in 53\% at EPS while $27 \%$ of borderline probands had sustained VT at EPS. The phenotype for ARVC/D develops over time and there is a potential for variation in the diagnostic accuracy of various tests that might change over time. (42) The finding that there were a similar frequency of desmosomal abnormalities of those phenotyped borderline and affected illustrates the relative insensitivity of the task force criteria for the diagnosis of early disease.

The study has several limitations. Not all diagnostic tests were done in every enrolled proband. Also in some patients, diagnostic studies were done prior to their being seen at the enrolling center. It was not feasible or practical to repeat these studies using the standardized protocols. Patients who had a cardioverter defibrillator implanted prior to referral to the enrolling center could not have a repeat MRI. Patients with probable desmosomal cardiomyopathy involving primarily the left ventricle were not referred for enrollment because the task force criteria exclude patients with moderate LV impairment.

In conclusion, the clinical profile of 108 newly diagnosed patients with suspected ARVC/D indicates that a combination of diagnostic imaging tests is needed to evaluate the presence of right ventricular structural, functional and electrical abnormalities. Echocardiography, RV angiography, SAECG and Holter monitoring provide optimal clinical evaluation of patients suspected of ARVC/D. In the early stages of ARVC/D, overall right ventricular function may be normal with local or regional wall motion abnormalities, and these are difficult to quantify.

\section{Supplementary Material}

Refer to Web version on PubMed Central for supplementary material.

\section{Acknowledgments}

Funding Support: NIH UO1-HL65594

\section{References}

1. Marcus FI, Fontaine G. Arrhythmogenic right ventricular dysplasia/cardiomyopathy, a review. Pacing Clin Electrophysiol 1995;18:1298-1314. [PubMed: 7659585]

2. Kies P, Bootsma M, Bax J, et al. Arrhythmogenic right ventricular dysplasia/cardiomyopathy: Screening, diagnosis, and treatment. Heart Rhythm 2006;3:225-234. [PubMed: 16443541]

3. Sen-Chowdhry S, Lowe MD, Sporton SC, et al. Arrhythmogenic right ventricular cardiomyopathy: clinical presentation, diagnosis, and management. Am. J.Medicine 2004;117:685-695. 
4. Thiene G, Nava A, Corrado D, et al. Right ventricular cardiomyopathy and sudden death in young people. N Engl J Med 1988;318:129-133. [PubMed: 3336399]

5. Maron BJ. Cardiovascular risks to young persons on the athletic field. Ann Int Med 1998;129:379386. [PubMed: 9735066]

6. Marcus, FI. Right ventricular dysplasia: Evaluation and management in relation to sports activities. In: Estes, NAM.; Salem, DN.; Wang, PJ., editors. Sudden Cardiac Death in the Athlete. Armonk, NY: Futura Publishing Co., Inc.; 1998. p. 277-284.

7. Corrado D, Basso C, Rizzoli G, et al. Does sports activity enhance the risk of sudden death in adolescents and young adults? J Am Coll Cardiol 2003;42:1959-1963. [PubMed: 14662259]

8. Marcus FI, Fontaine GH, Guiraudon G, et al. Right ventricular dysplasia: A report of 24 adult cases. Circulation 1982;65:384-399. [PubMed: 7053899]

9. Bomma C, Rutberg J, Tandri H, et al. Misdiagnosis of arrhythmogenic right ventricular dysplasia/ cardiomyopathy. J Cardiovasc Electrophysiol 2004;15:300-306. [PubMed: 15030420]

10. McKenna WJ, Thiene G, Nava A, et al. Diagnosis of Arrhythmogenic Right Ventricular Dysplasia/ Cardiomyopathy. Task Force of the Working Group Myocardial and Pericardial Disease of the European Society of Cardiology and of the Scientific Council on Cardiomyopathies of the International Society and Federation of Cardiology. Br. Heart J 1994;71:215-218. [PubMed: 8142187]

11. Marcus F, Towbin J, Zareba W, et al. Arrhythmogenic right ventricular dysplasia/cardiomyopathy (ARVD/C) a multidisciplinary study: Design and protocol. Circulation 2003;107:2975-2978. [PubMed: 12814984]

12. Nava AF, Folino F, Bauce B, et al. Signal-averaged electrocardiogram in patients with arrhythmogenic right ventricular cardiomyopathy and ventricular arrhythmias. Eur Heart J 2000;21:58-65. [PubMed: 10610745]

13. Buxton AE, Fisher JD, Josephson ME, et al. Prevention of sudden death in patients with coronary artery disease: the Multicenter Unsustained Tachycardia Trial (MUSTT). Prog in Cardiovasc Dis 1993;36:215-226.

14. Yoerger DM, Marcus F, Sherrill D, et al. Echocardiographic findings in patients meeting task force criteria for arrhythmogenic right ventricular dysplasia. J Am Coll Cardiol 2005;45:860-865. [PubMed: 15766820]

15. Tandri H, Friedrich MG, Calkins H, et al. MRI of arrhythmogenic right ventricular cardiomyopathy/ dysplasia. J Cardiovasc Magn Res 2004;6:557-563.

16. Basso, C.; Thiene, G. Autopsy and endomyocardial biopsy findings. In: Marcus, F.; Nava, A.; Thiene, G., editors. Arrhythmogenic RV Cardiomyopathy/Dysplasia. Springer-Verlag Italia: 2007. p. 29-44.

17. Basso C, Ronco F, Marcus F, et al. Quantitative assessment of endomyocardial biopsy in arrhythmogenic right ventricular cardiomyopathy/dysplasia: an in vitro validation of diagnostic criteria. Eur Heart J 2008;29:2760-2771. [PubMed: 18819962]

18. Dalal D, Nasir K, Bomma C, et al. Arrhythmogenic right ventricular dysplasia A United States experience. Circulation 2005;112:3823-3832. [PubMed: 16344387]

19. Heidbuchel H, Hoogsteen J, Fagard R, et al. High prevalence of right ventricular involvement in endurance athletes with ventricular arrhythmias. Role of an electrophysiologic study in risk stratification. Eur Heart J 2003;24:1473-1480. [PubMed: 12919770]

20. Marcus F, Towbin JA. The mystery of arrhythmogenic right ventricular dysplasia/cardiomyopathy: from observation to mechanistic explanation. Circulation 2006;114:1794-1795. [PubMed: 17060394]

21. Kirchhof P, Fabritz L, Zwiener M, et al. Age and training dependent development of arrhythmogenic right ventricular cardiomyopathy in heterozygous plakoglobin deficient mice. Circulation 2006;113:1799-1806. [PubMed: 16606801]

22. Peters S, Trummel M. Diagnosis of arrhythmogenic right ventricular dysplasia/cardiomyopathy: Value of standard ECG revisited. A.N.E 2003;8:238-245. [PubMed: 14510660]

23. Nasir K, Bomma C, Tandri H, et al. Electrocardiographic features of arrhythmogenic right ventricular dysplasia/cardiomyopathy according to disease severity. A need to broaden diagnostic criteria. Circulation 2004;110:1527-1534. [PubMed: 15381658] 
24. Cox MGPJ, Nelen MR, Wilde AAM, et al. Activation delay and VT parameters in arrhythmogenic right ventricular dysplasia/cardiomyopathy: Toward improvement of diagnostic ECG criteria. J Cardiovasc Electrophysiol 2008;19:775-781. [PubMed: 18373594]

25. Bauce B, Basso C, Rampazzo A, et al. Clinical profile of four families with arrhythmogenic right ventricular cardiomyopathy caused by dominant desmoplakin mutations. Eur Heart J 2005;26:16661675. [PubMed: 15941723]

26. Sen-Chowdhry S, Syrris P, Ward D, et al. Clinical and genetic characterization of families with arrhythmogenic right ventricular dysplasia/cardiomyopathy provides novel insights into patterns of disease expression. Circulation 2007;115:1710-1720. [PubMed: 17372169]

27. Sen-Chowdhry S, Syrris P, Prasad SK, et al. Left-dominant arrhythmogenic cardiomyopathy: an under-recognized clinical entity. J Am Coll Cardiol 2008;52:2175-2187. [PubMed: 19095136]

28. Sievers B, Addo M, Franken U, et al. Right ventricular wall motion abnormalities found in healthy subjects by cardiovascular magnetic resonance imaging and characterized with a new segmental model. J Cardiovasc Magn Res 2004;6:601-608.

29. Bluemke DA, Krupinski EA, Ovitt T, et al. MR Imaging of arrhythmogenic right ventricular cardiomyopathy: Morphologic findings and interobserver reliability. Cardiology 2003;99:153-162. [PubMed: 12824723]

30. Burke AP, Farb A, Tashko G, et al. Arrhythmogenic right ventricular cardiomyopathy and fatty replacement of the right ventricular myocardium: are they different diseases? Circulation 1998;97:1571-1580. [PubMed: 9593562]

31. Fontaine G, Fontaliran F, Zenati O, et al. Fat in the heart. A feature unique to the human species? Observational reflections on an unsolved problem. Acta Cardiologica 1999;54:189-194. [PubMed: 10511894]

32. Maceira AM, Prasad SK, Khan M, et al. Reference right ventricular systolic and diastolic function normalized to age, gender and body surface area from steady-state free precession cardiovascular magnetic resonance. Eur Heart J 2006;27:2879-2888. [PubMed: 17088316]

33. Tandri H, Daya SK, Nasir K, et al. Normal reference values for the adult right ventricle by magnetic resonance imaging. Am J Cardiol 2006;98:1660-1664. [PubMed: 17145230]

34. Indik JH, Dallas WJ, Ovitt T, et al. Do patients with right ventricular outflow ventricular arrhythmias have normal right ventricular wall motion? A quantitative analysis compared to normal subjects. Cardiology 2005;104:10-15. [PubMed: 15942177]

35. Bowles N, Ni J, Marcus F, et al. The detection of cardiotropic viruses in the myocardium of patients with arrhythmogenic right ventricular dysplasia/cardiomyopathy. J Am Coll Cardiol 2002;39:892895. [PubMed: 11869858]

36. Calabrese F, Angelini A, Thiene G, et al. No detection of enteroviral genome in the myocardium of patients with arrhythmogenic right ventricular cardiomyopathy. J Clin Pathol 2000;53:382-387. [PubMed: 10889821]

37. Fontaliran F, Fontaine G, Brestescher C, et al. Signification del infiltrates lymphoplasmocytaires dans la dyplasia ventriculare droite arhythmogene. Arch Mal Coeur 1995;88:1021-1028. [PubMed: 7487318]

38. Basso C, Thiene G, Corrado D, Angelini A, Nava A, Valente M. Arrhythmogenic right ventricular cardiomyopathy: dysplasia, dystrophy or myocarditis? Circulation 1996;94:983-991. [PubMed: 8790036]

39. Chimenti C, Pieroni M, Maseri A, et al. Histologic findings in patients with clinical and instrumental diagnosis of sporadic arrhythmogenic right ventricular dysplasia. J Am Coll Cardiol 2004;43:23052313. [PubMed: 15193698]

40. Wichter T, Hindricks G, Lerch H, et al. Regional myocardial sympathetic dysinnervation in arrhythmogenic right ventricular cardiomyopathy. An analysis using I-meta-iodobenzylguanidine scintigraphy. Circulation 1994;89:667-683. [PubMed: 8313555]

41. Ott P, Marcus F, Sobonya RE, et al. Cardiac sarcoidosis masquerading as right ventricular dysplasia. PACE 2003:1498-1503. [PubMed: 12914628]

42. Kies P, Bootsma M, Bax JJ, et al. Serial reevaluation for ARVD/C is indicated in patients presenting with left bundle branch block ventricular tachycardia and minor ECG abnormalities. J Cardiovasc Electrophysiol 2006;17:586-593. [PubMed: 16836703] 


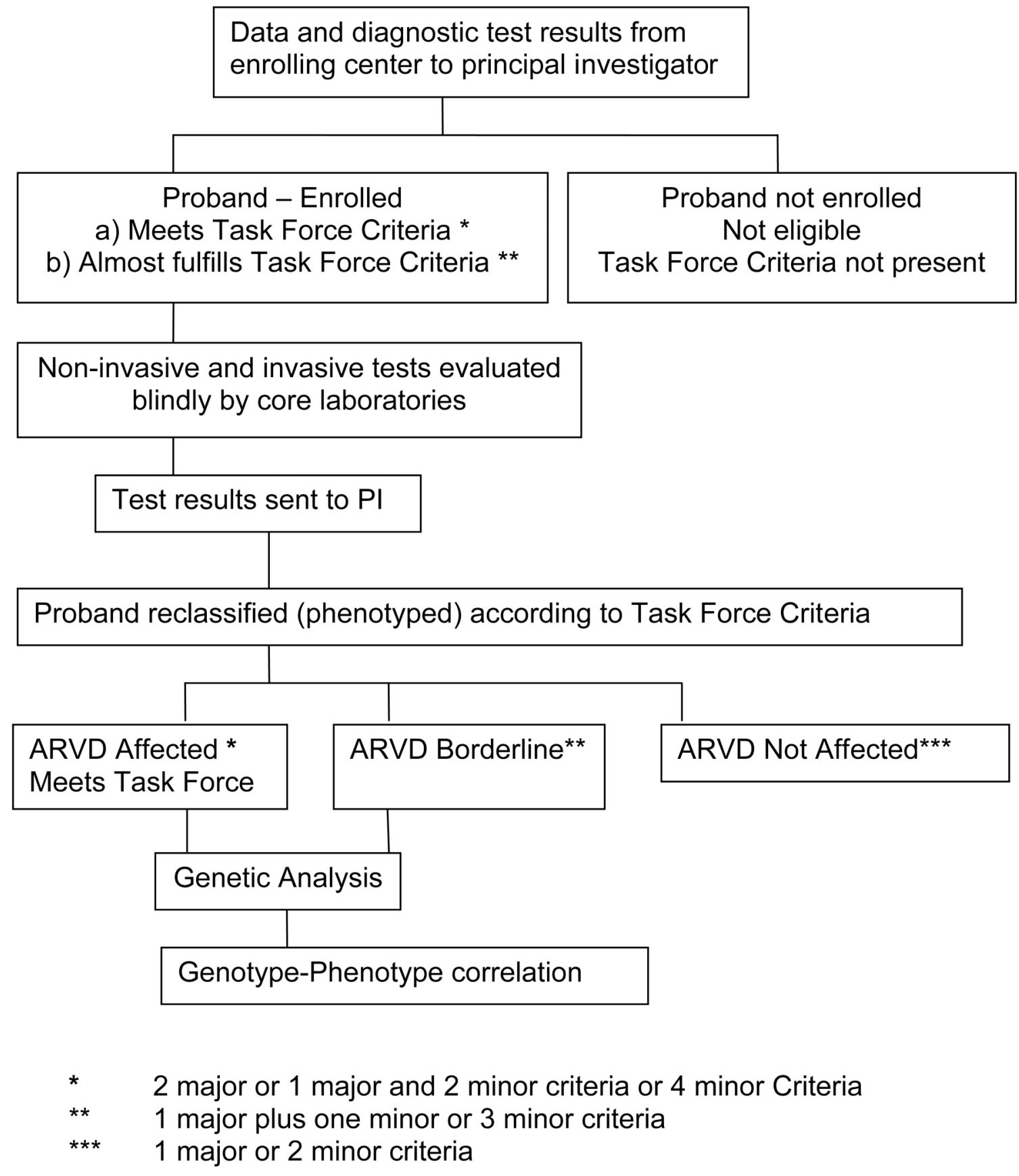

Figure 1. 


\section{Figure 2A}

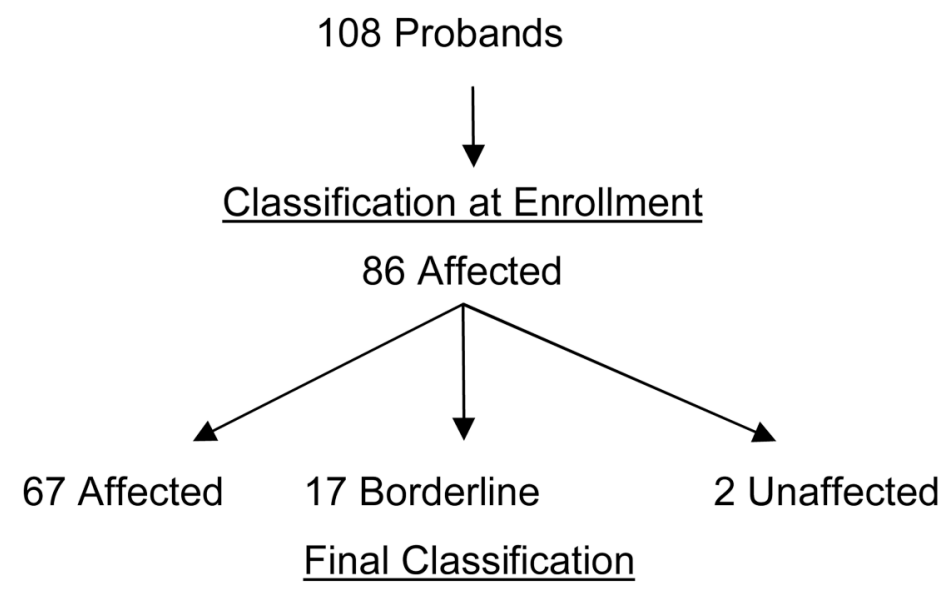

Figure 2B

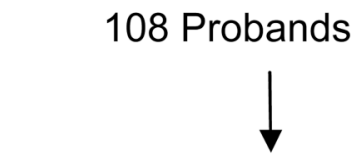

Classification at Enrollment

19 Borderline

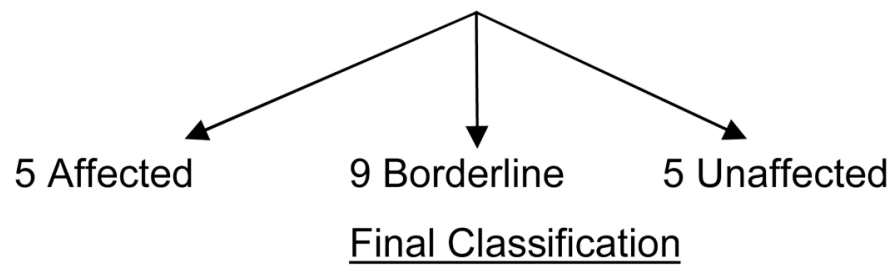

Figure 2.

Three additional probands were classified as suspicious (one major criteria or two minor criteria) at enrollment and had final classification as affected $(n=1)$, and borderline $(n=2)$. 


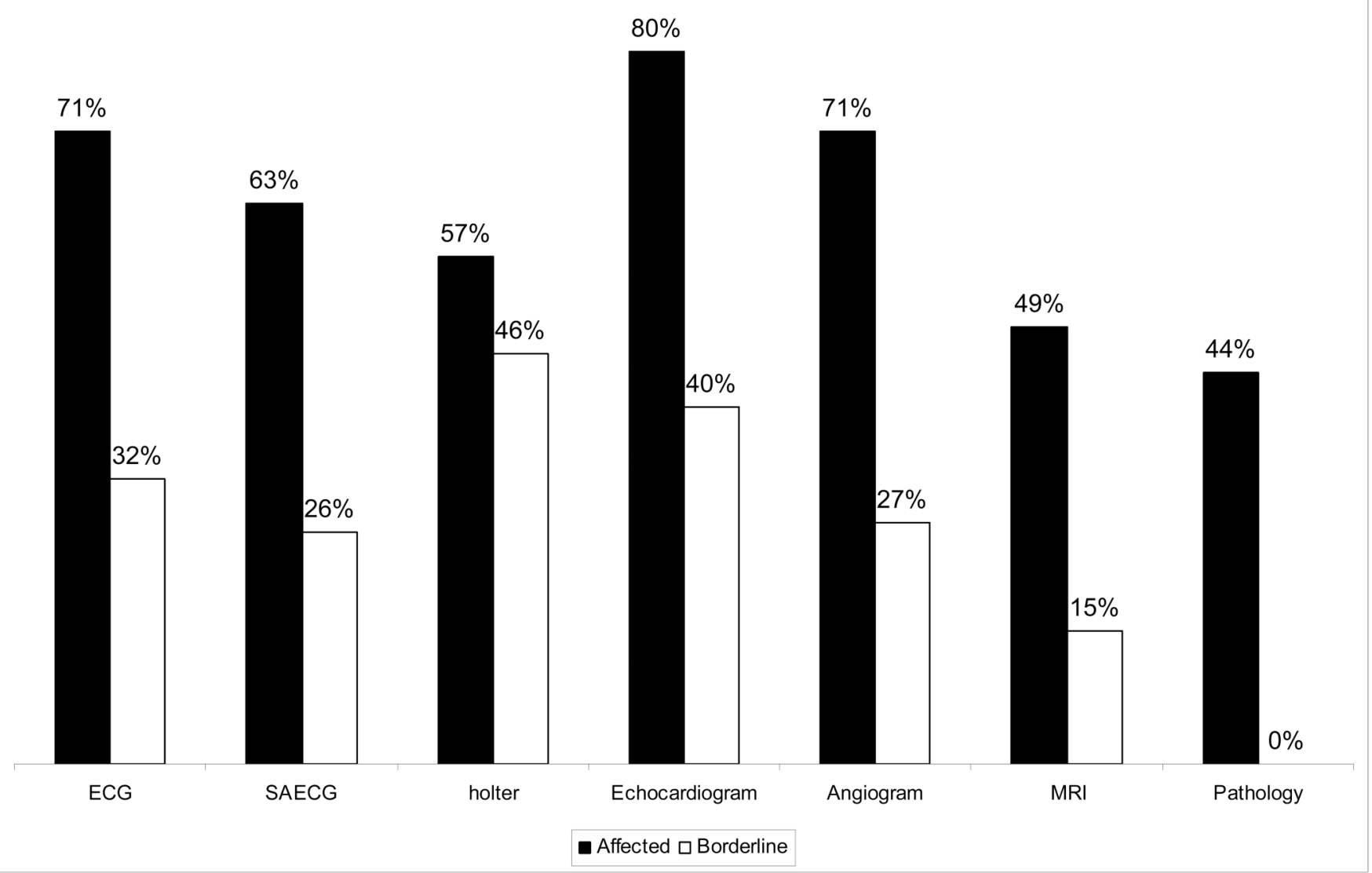

Figure 3. 


\section{C-Statistics Decline}

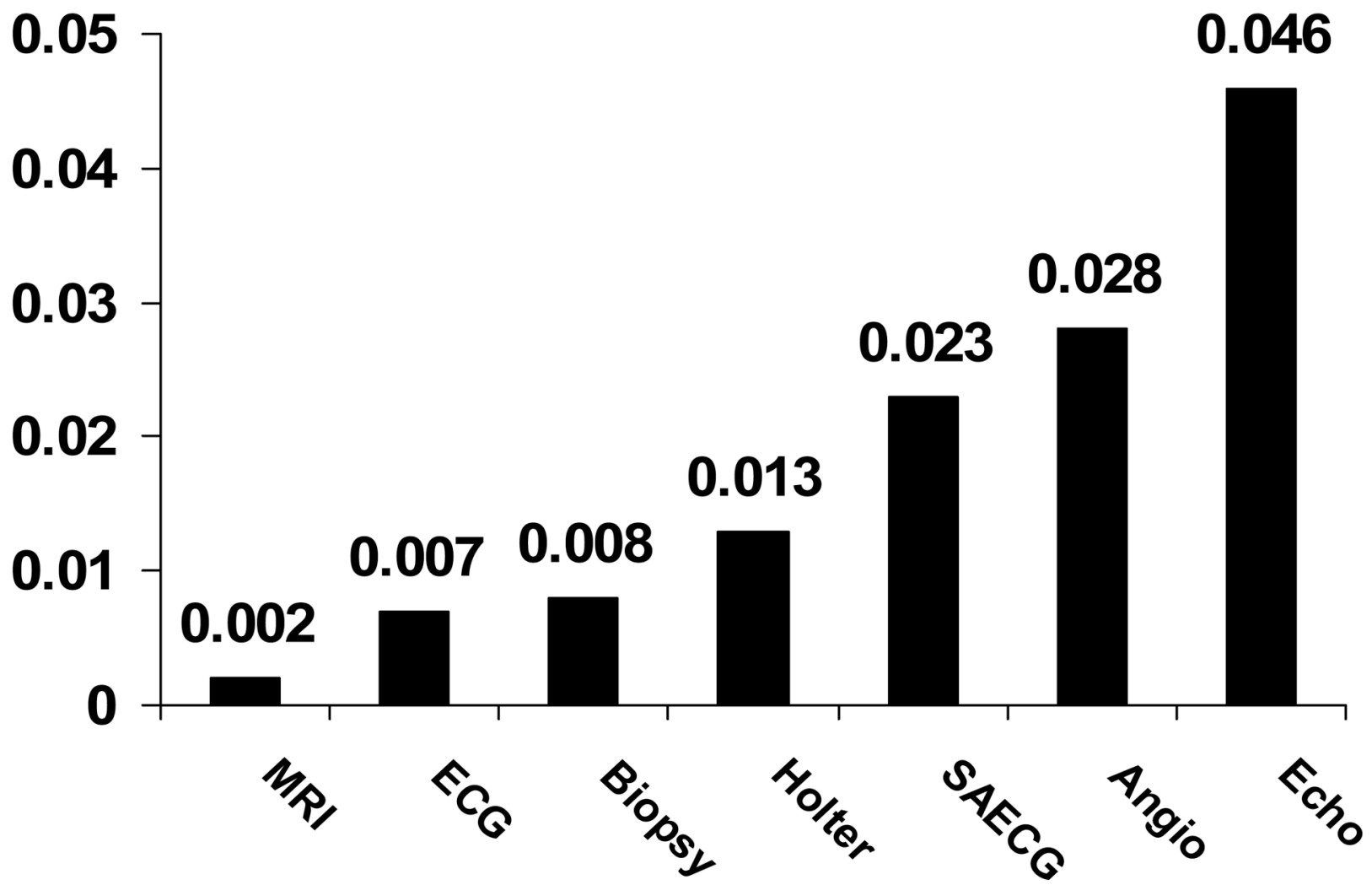

Figure 4. 
Table 1

Clinical and ECG findings in the 108 probands

\begin{tabular}{|c|c|c|}
\hline Age onset of symptoms & & \\
\hline Age at diagnosis & & \\
\hline Age at enrollment & & \\
\hline Sex ratio $(\mathrm{m} / \mathrm{f})$ & \multicolumn{2}{|c|}{$62 / 46$} \\
\hline Symptoms & $\mathrm{N}=108$ & $\%$ \\
\hline Palpitations & 61 & 56 \\
\hline Dizziness & 29 & 27 \\
\hline Syncope & 23 & 21 \\
\hline Chest pain & 15 & 14 \\
\hline 12 lead ECGs & $\mathrm{N}=95^{*}$ & \\
\hline No TWI [11A,8B,5U] & 24 & 25 \\
\hline TWI only V1 [11a,5B,1U] & 17 & 18 \\
\hline TWI only V1 - V2 [3A,3B] & 6 & 6 \\
\hline TWI only V1 - V3 [11A,4B] & 15 & 16 \\
\hline TWI extending beyond V3 [29A,1B] & 30 & 32 \\
\hline TWI not in V2 and V3 but beyond & 3 & 3 \\
\hline QRS prolongation > 110 msecs in $\mathrm{V} 1, \mathrm{~V} 2$, and $\mathrm{V} 3[4 \mathrm{~A}, 1 \mathrm{U}]$ & 5 & 5 \\
\hline QRS prolongation in $\mathrm{V} 1-\mathrm{V} 3 / \mathrm{V} 4-\mathrm{V} 6>1.2[12 \mathrm{~A}, 3 \mathrm{~B}, 1 \mathrm{U}]$ & 16 & 17 \\
\hline $\mathrm{S}$ wave duration $\geq 55$ msecs $[18 \mathrm{~A}, 11 \mathrm{~B}, 2 \mathrm{U}]$ & 31 & 32 \\
\hline Signal averaged ECG & $\mathrm{N}=86$ & \\
\hline Late potentials ( 2 of 3 criteria abnormal) & $50^{*}$ & 58 \\
\hline Holter monitor & $\mathrm{N}=86$ & \\
\hline PVCs $>1000 / 24$ hours & 49 & 57 \\
\hline Sustained VT clinical & $\mathrm{N}=38^{* *}$ & 35 \\
\hline LBBB sup VT & 14 & \\
\hline LBBB inf VT & 10 & \\
\hline LBBB indeterminate axis & 8 & \\
\hline VT of indeterminate morphology & 5 & \\
\hline RBBB & 1 & \\
\hline Electrophysiology study performed & $\mathrm{N}=75$ & \\
\hline Sustained VT at EPS & $\mathrm{N}=36^{* *}$ & 49 \\
\hline LBBB sup VT & 15 & \\
\hline LBBB inf VT & 15 & \\
\hline LBBB indeterminate axis & 3 & \\
\hline RBBB & 3 & \\
\hline Histopathology of endomyocardial biopsy & $\mathrm{N}=64$ & \\
\hline Meet histomorphometric criteria & 20 & 31 \\
\hline Familial history & & \\
\hline Familial history of ARVC/D & 23 & 21 \\
\hline Family disease confirmed at autopsy & 6 & 6 \\
\hline Sudden death in family member age $<35$ years & 8 & 7 \\
\hline
\end{tabular}


Numbers exclude missing data

* Excluding 11 RBBB, 1 LBBB, 1 uninterpretable (ventricular paced)

*** Report or documented by ECG

TWI T wave inversion, LBBB Left bundle branch block, RBBB Right bundle branch block Final phenotype as A=Affected, B=Borderline, U=Unaffected 


\section{Table 2}

Comparison of RV function and wall motion abnormalities reported by the core laboratories for 2D echocardiogram, MRI and angiography in studies adequate for interpretation of RVEF, FAC and wall motion

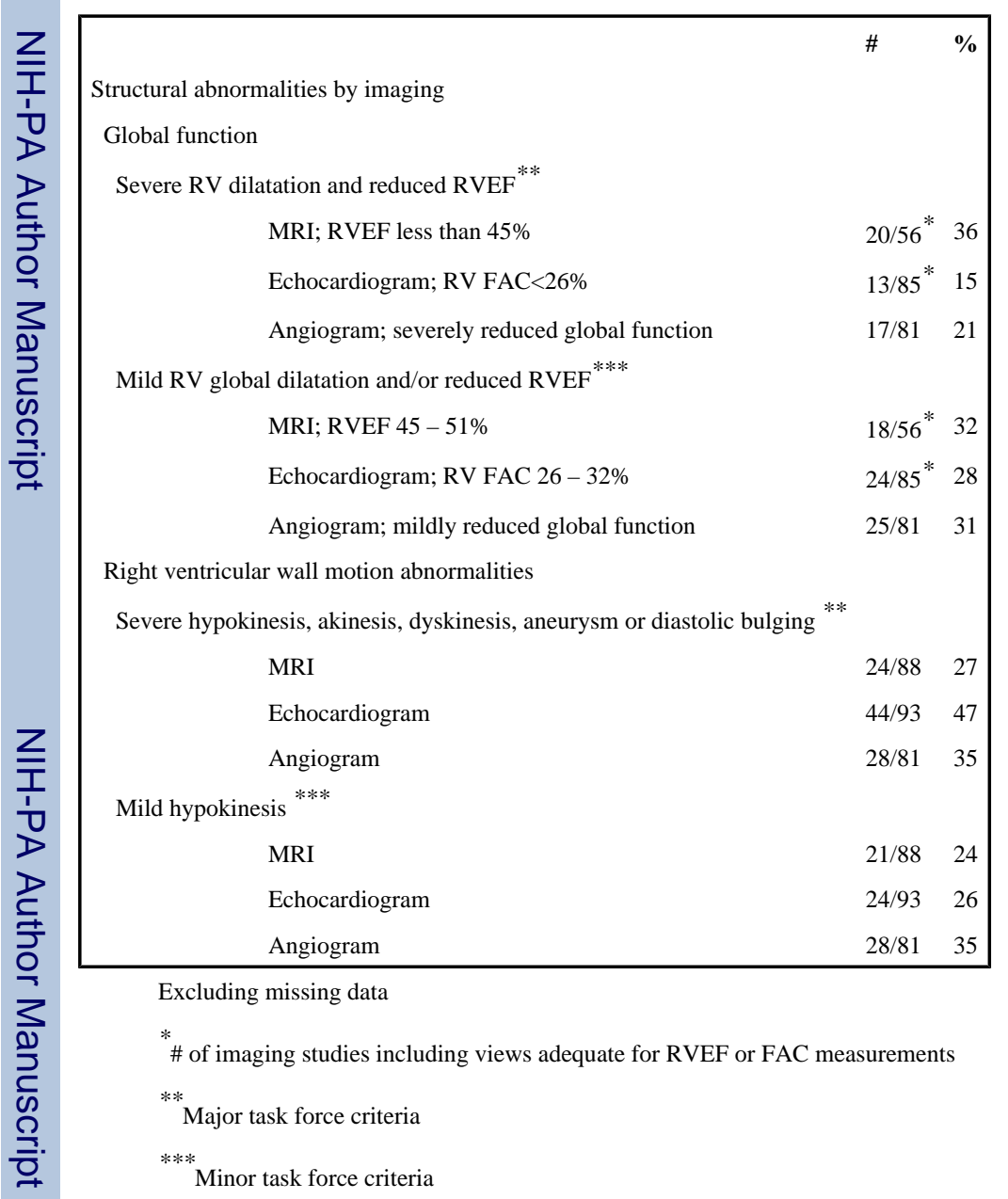




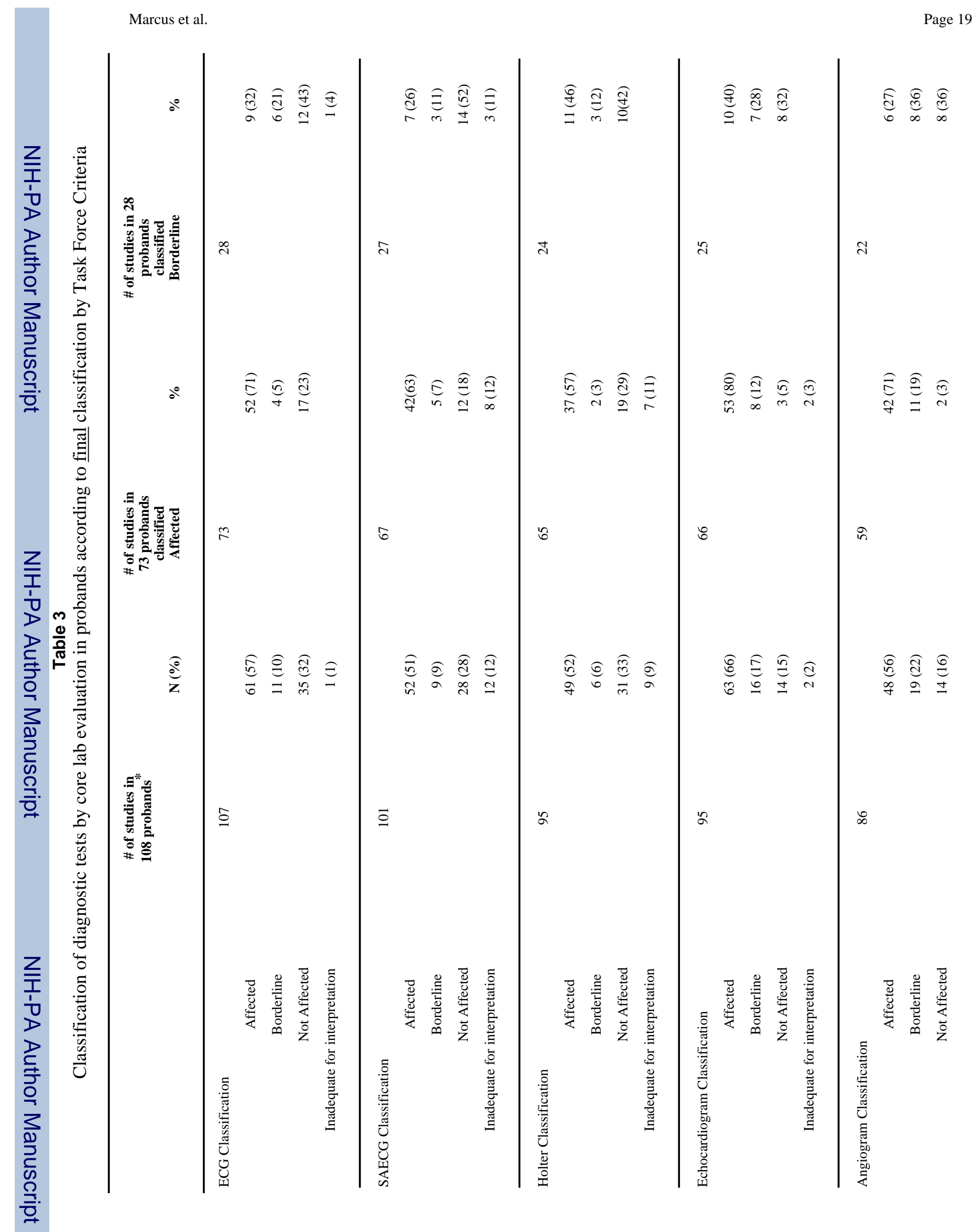

Heart Rhythm. Author manuscript; available in PMC 2010 July 1. 


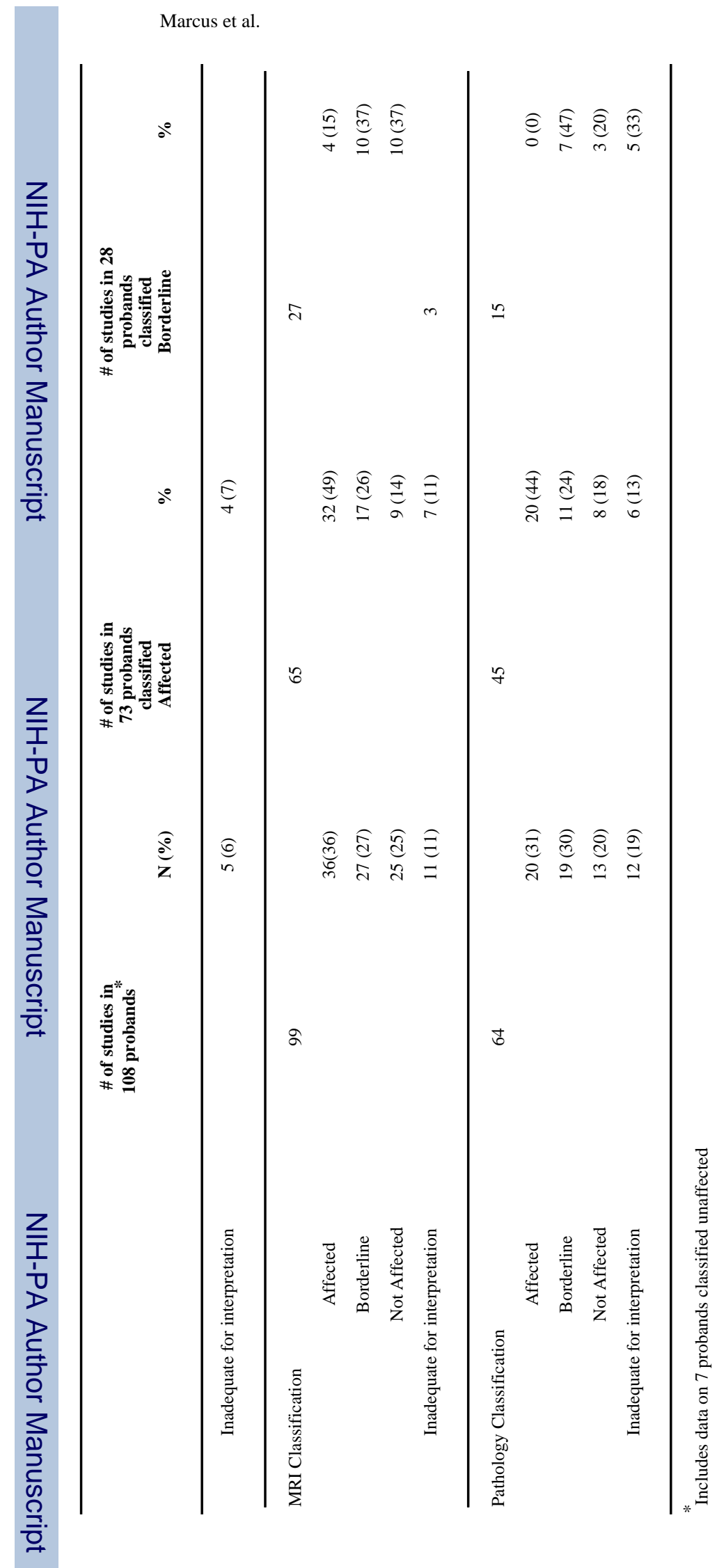

Heart Rhythm. Author manuscript; available in PMC 2010 July 1. 
Table 4

Comparison of Predictive Models with Fewer than 7 Tests for Correct Classification of ARVC/D.

\begin{tabular}{|c|c|c|c|}
\hline $\begin{array}{l}\text { Number of } \\
\text { Variables } \\
\text { In the Model }\end{array}$ & $\begin{array}{l}\text { Variables Included } \\
\text { in the Predictive Model }\end{array}$ & $\begin{array}{l}\text { Variables } \\
\text { Removed }\end{array}$ & $\begin{array}{c}\text { Score } \\
\text { Chi-Square }\end{array}$ \\
\hline 7-Variable Model & $\begin{array}{c}\text { Echo, Angio, SAECG, Holter, } \\
\text { Biopsy, ECG, MRI }\end{array}$ & None & 61.44 \\
\hline $\begin{array}{l}\text { Best 6-Variable } \\
\text { Model }\end{array}$ & $\begin{array}{c}\text { Echo, Angio, SAECG, Holter, } \\
\text { Biopsy, ECG }\end{array}$ & MRI & 61.24 \\
\hline $\begin{array}{l}\text { Best 5-Variable } \\
\text { Model }\end{array}$ & $\begin{array}{c}\text { Echo, Angio, SAECG, Holter, } \\
\text { ECG }\end{array}$ & Biopsy, MRI & $59.34^{*}$ \\
\hline $\begin{array}{l}\text { Best 4-Variable } \\
\text { Model }\end{array}$ & Echo, Angio, SAECG, Holter & $\begin{array}{l}\text { ECG, Biopsy, } \\
\text { MRI }\end{array}$ & $57.11^{*}$ \\
\hline $\begin{array}{l}\text { Best 3-Variable } \\
\text { Model }\end{array}$ & Echo, Angio, SAECG & $\begin{array}{l}\text { Holter, Biopsy, } \\
\text { ECG, MRI }\end{array}$ & $52.26^{*}$ \\
\hline
\end{tabular}

